\title{
Examination of pulse oximetry tracings to detect obstructive sleep apnea in patients with advanced chronic obstructive pulmonary disease
}

\author{
Adrienne S Scott MSc ${ }^{1}$, Marcel A Baltzan MSc MD ${ }^{1,2}$, Norman Wolkove MD ${ }^{1}$
}

\begin{abstract}
AS Scott, MA Baltzan, N Wolkove. Examination of pulse oximetry tracings to detect obstructive sleep apnea in patients with advanced chronic obstructive pulmonary disease. Can Respir J 2014;21(3):171-175.

Nocturnal hypoxemia and obstructive sleep apnea (OSA) are common comorbidities in patients with chronic obstructive pulmonary disease (COPD). The authors sought to develop a strategy to interpret nocturnal pulse oximetry and assess its capacity for detection of OSA in patients with stage 3 to stage 4 COPD. A review of consecutive patients with COPD who were clinically prescribed oximetry and polysomnography was conducted. OSA was diagnosed if the polysomnographic apnea-hypopnea index was $>15$ events/h. Comprehensive criteria were developed for interpretation of pulse oximetry tracings through iterative validation and interscorer concordance of $\geq 80 \%$. Criteria consisted of visually identified desaturation 'events' (sustained desaturation $\geq 4 \%, 1 \mathrm{~h}$ time scale), 'patterns' ( $\geq 3$ similar desaturation/saturation cycles, $15 \mathrm{~min}$ time scale) and the automated oxygen desaturation index. The area under the curve (AUC), sensitivity, specificity and accuracy were calculated. Of 59 patients (27 male), 31 had OSA (53\%). The mean forced expiratory volume in $1 \mathrm{~s}$ was $46 \%$ of predicted (range $21 \%$ to $74 \%$ of predicted) and $52 \%$ of patients were on long-term oxygen therapy. Among 59 patients, 35 were correctly identified as having OSA or not having OSA, corresponding to an accuracy of $59 \%$, with a sensitivity and specificity of $59 \%$ and $60 \%$, respectively. The AUC was 0.57 (95\% CI 0.55 to 0.59 ). Using software-computed desaturation events (hypoxemia $\geq 4 \%$ for $\geq 10$ s) indexed at $\geq 15$ events/h of sleep as diagnostic criteria, sensitivity was $60 \%$, specificity was $63 \%$ and the AUC was 0.64 (95\%CI 0.62 to 0.66 ). No single criterion demonstrated important diagnostic utility. Pulse oximetry tracing interpretation had a modest diagnostic value in identifying OSA in patients with moderate to severe COPD.
\end{abstract}

Key Words: Chronic obstructive pulmonary disease; Nocturnal hypoxemia; Obstructive sleep apnea; Oximetry

T has been reported that sleep-related respiratory changes normally occur in healthy individuals due to factors such as decreased metabolic rate, decreased chemosensitivity, decreased ventilation and increased air resistance, and are considered to be part of the normal respiratory physiology of sleep (1). These changes are most pronounced during rapid eye movement sleep and can have a negative impact on blood gas tensions and oxygen saturation $(1,2)$. In advanced chronic obstructive pulmonary disease (COPD), these normal physiological phenomena in sleep can have adverse effects due, in part, to compromised mechanical configuration of the diaphragm as well as chest wall and ventilation-perfusion mismatch (3). Nocturnal hypoxemia can be severe in patients with COPD due to upper airway obstruction typical of obstructive sleep apnea (OSA) combined with hypoventilation due to obesity, COPD or both. The presence of nocturnal oxygen desaturation is associated with cardiovascular risks (fatal and nonfatal) as well as the evolution of pulmonary hypertension $(4,5)$. For these reasons, although the prevalence of OSA in COPD is similar to that of the general population, it is the overlap of both conditions that creates a greater opportunity for comorbidity and a negative prognosis (6). Currently, a clinical diagnosis of OSA is made after a patient undergoes polysomnography (PSG). Polysomnographic sleep
L'examen des tracés de saturométrie pour dépister

l'apnée obstructive du sommeil chez les patients atteints de maladie pulmonaire obstructive chronique avancée

L'hypoxémie nocturne et l'apnée obstructive du sommeil (AOS) sont des comorbidités courantes chez les patients atteints de maladie obstructive chronique (MPOC). Les auteurs ont cherché à élaborer une stratégie pour interpréter la saturométrie nocturne et en évaluer la capacité de dépister l'AOS chez les patients atteints de MPOC de stade 3 à 4 . Ils ont effectué une analyse des patients consécutifs atteints de MPOC à qui on avait prescrit une oxymétrie et une polysomnographie en clinique. L'AOS était diagnostiquée si l'indice polysomnographique apnée-hypopnée était supérieur à 15 événements à l'heure. Les auteurs ont établi des critères détaillés pour interpréter les tracés de saturométrie par validation itérative et concordance interévaluateur d'au moins $80 \%$. Ces critères consistaient à déterminer visuellement les « événements » de désaturation (désaturation soutenue d'au moins $4 \%$, échelle d'une heure), les "profils " (au moins trois cycles similaires de désaturation/saturation, échelle de 15 minutes) et la mesure automatique de l'indice de désaturation en oxygène. Ils en ont calculé l'aire sous la courbe (ASC), la sensibilité, la spécificité et la précision. Sur 59 patients (27 de sexe masculin), 31 faisaient de l'AOS (53\%). Le volume expiratoire maximal moyen par seconde était de $46 \%$ (plage de $21 \%$ à $74 \%$ ) de la valeur prévue, et $52 \%$ des patients étaient sous oxygénothérapie à long terme. Chez ces 59 patients, les auteurs ont établi correctement que 35 faisaient ou non de l'AOS, pour une exactitude de $59 \%$, une sensibilité de $59 \%$ et une spécificité de $60 \%$. L'ASC était de 0,57 (95\% IC 0,55 à 0,59). Après avoir indexé dans les critères diagnostiques les événements de désaturation informatisés (hypoxémie d'au moins $4 \%$ pendant au moins dix secondes) à au moins 15 événements par heure de sommeil, la sensibilité s'élevait à $60 \%$, la spécificité, à $63 \%$, et l'ASC, à 0,64 (95\% IC 0,62 à 0,66). Aucun critère unique n'en démontrait une utilité diagnostique importante. L'interprétation du tracé de saturométrie avait une valeur diagnostique modeste pour dépister l'AOS chez les patients atteints de MPOC modéré à grave.

studies are costly, usually require attended monitoring and are of limited availability in some regions. Clinicians do not have a standardized strategy for selecting patients for PSG when OSA is suspected using nocturnal oximetry because there are few physiological markers recorded in this procedure that have been found to be reliable predictors of a patient with advanced COPD to be also likely diagnosed with OSA. Nevertheless, finding and treating OSA in patients with advanced COPD, including those on supplemental oxygen, is important. Patients who comply with treatment have a higher survival rate than those who are not treated (7).

Nocturnal pulse oximetry to assess oxygen saturation patterns throughout the usual sleep period has been extensively studied in the diagnostic evaluation of patients typically encountered in sleep clinics, is relatively simple to execute and is, as a result, inexpensive. Continuous oximetry monitoring has also been used to evaluate the profiles of patients with advanced COPD including respiratory failure. The objective of the present study was to develop criteria for visual inspection by nonexpert scorers to interpret nocturnal pulse oximetry tracings and assess its capacity for detection of OSA in patients with advanced COPD.

${ }^{1}$ Mount Sinai Hospital Centre; ${ }^{2}$ Department of Epidemiology and Biostatistics, McGill University, Montreal, Quebec

Correspondence: Ms Adrienne S Scott, Research office C1.20, Mount Sinai Hospital Centre, 5690 Cavendish Boulevard, Cote St-Luc, Montreal, Quebec H4W 1S7. Telephone 514-369-2222 ext 1060, fax 514-369-2225, e-mail recherche.sinai@ssss.gouv.qc.ca 


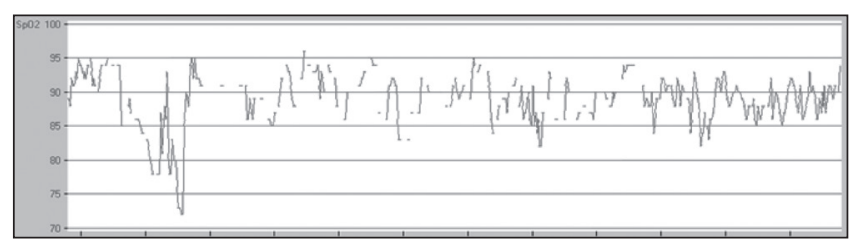

Figure 1) An example of an excluded oximetry tracing with time frame of $1 \mathrm{~h}$

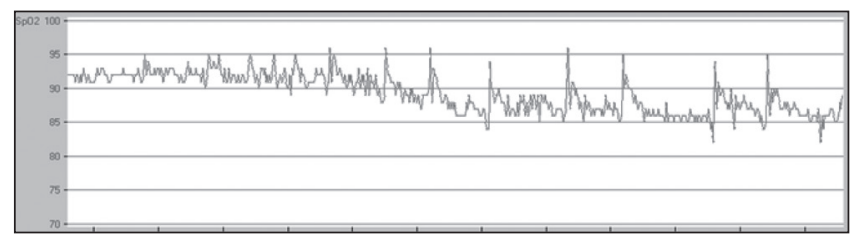

Figure 2) An example of a desaturation 'event' from an oxygen saturation oximetry tracing with a time frame of $1 \mathrm{~h}$

\section{METHODS}

Protocol

A consecutive chart review of the inpatient pulmonary rehabilitation service from January 1, 2006 to November 1, 2010 at Mount Sinai Hospital (Montreal, Quebec) was conducted. Patients were electively admitted for three to four weeks for pulmonary rehabilitation to improve functional capacity. Stable COPD was defined in patients who did not experience an exacerbation over a period of six weeks. Patients were identified and included if medical records' coding for COPD, overnight pulse oximetry and PSG were present. This included patients who were undergoing long-term oxygen therapy (LTOT). The present study was approved by the Mount Sinai Hospital Ethics Committee.

With overnight PSG, the presence or absence of OSA was determined at a prespecified apnea/hypopnea index (AHI) cut-off of $>15$ events/h sleep. PSG recording and scoring followed the current standards of the American Academy of Sleep Medicine (AASM) (8) and used software for manual analysis of digital files (Sandman, Tyco, Canada and Nihon Kohden America, USA) that included the standard recordings of electro-oculogram, electroencephalogram, electrocardiogram and submental electromyography, with concurrent monitoring of respiratory variables during sleep including thermistor and nasal pressure waves, pulse oximetry and inductance plethysmography (9). Apneas were scored with at least $10 \mathrm{~s}$ of a $90 \%$ reduction in airflow according to thermistor. Hypopneas were scored using the official AASM alternative criteria when accompanied by at least a 3\% desaturation or electroencephalogram arousal to avoid false-negative polysomnographic diagnoses of OSA in patients with a low body mass index or short times with sleep in the supine position. The overnight pulse oximetry (Respironics 920N + Oximeter, Respironics, USA) procedures were performed on a separate night within two weeks using a transcutaneous ear probe for a minimum of six continuous hours while the patient slept in his/her hospital bed. This procedure was repeated if sleep was observed to be disturbed by patient or environmental factors, or if the recording was of poor quality as determined by the care team. An electronic recording (ProFox Oximetry Standard 0103.12S, Respironics, USA) was then produced with the following automated analysis outcomes: mean oxygen saturation $\left(\mathrm{SpO}_{2}\right)$, highest/lowest $\mathrm{SpO}_{2}$, oxygen desaturation events index (ODI); desaturation event (a desaturation of $\geq 4 \%$ for $\geq 10 \mathrm{~s}$ and $\leq 3 \mathrm{~min} /$ desaturation events per sampled hour), mean high/low desaturation, and the percentage of recorded time spent with an oxygen saturation $<90 \%$ (\% time $\mathrm{SpO}_{2}$ $<90$ ). The sampling frequency of the oximeter was $1 \mathrm{~Hz}$ on memory interval and an averaging time of $3 \mathrm{~s}$. Pulse oximetry visual analysis for recognition of prespecified patterns was performed prospectively for the purposes of the present research using commercially available software (ProFox Oximetry Standard 0103.12S).

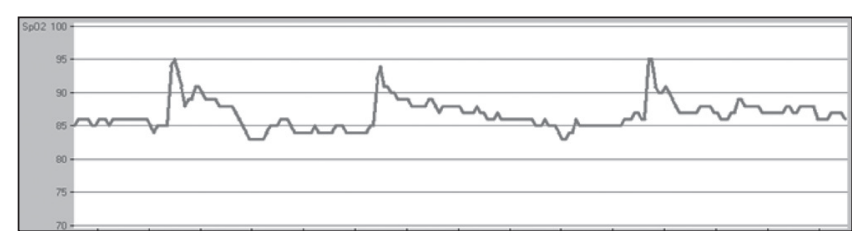

Figure 3) An example of an oxygen saturation 'pattern' from an oximetry tracing with a time frame of $15 \mathrm{~min}$

\section{Oximetry tracing interpretation protocol}

After review of the literature on the waveforms used to identify OSA with oximetry, a comprehensive set of criteria was developed for interpretation of pulse oximetry tracings through iterative validation and interscorer concordance $\geq 80 \%$ (author AS and acknowledged RC). Criteria consisted of quantification of visually identified desaturation 'events' (continuous desaturation $\geq 4 \%$ lasting $\geq 30 \mathrm{~min}$, viewed in a $2 \mathrm{~h}$ time scale window), saturation 'patterns' ( $\geq 3$ similar desaturation/ saturation cycles, in a 15 min time scale window) and mean saturation within each pattern was reported (Appendix 1). Using this method, a patient is identified to have OSA if the following criteria are met: A mean $\mathrm{SpO}_{2} \leq 90 \%$ and at least one of the following - number of events $\geq 4$ and/or number of patterns $\geq 12$. Both 'events' and 'patterns' could coexist in the same tracing. To determine whether more or fewer events or patterns influenced the diagnostic accuracy, further ROC analysis was performed for each component of the interpretation criteria as well as the automated ODI provided by the software counting $4 \%$ desaturations. The oximetry tracings were scored blind of the PSG results. These findings were then prospectively compared with the PSG diagnosis. Examples of an excluded oximetry recording are shown in Figure 1, in which $\geq 20 \%$ of the total tracing time contains breaks (ie, a desaturation 'event' [Figure 2]), whereby an average desaturation of $\geq 4 \%$ from the baseline average was observed. The oxygen desaturation/ resaturation 'pattern' depicted in Figure 3 is marked by a rise and fall of at least three similar saturation cycles (see Appendix I for greater detail regarding tracing exclusion, desaturation event and pattern recognition).

\section{Statistical analysis}

Descriptive statistics (means, SDs, counts and frequencies in percent) were used to present patients' baseline characteristics. Differences between patients diagnosed with OSA and no OSA were tested using unpaired $t$ tests. Contingency analysis and calculation of ROC curves were performed to assess the detection capacity of various oximetry indexes to identify OSA. A web-based ROC calculator with an ordinal rating scale format was used for analyses (10). The ordinal rating scale format enabled the calculation of a confidence value (1 to 6) for ranges of AHI scores derived from PSG results such that a rating of $1=$ definitely no OSA (AHI range of 0 to 5 ) and $6=$ definitely OSA (AHI >30). The sensitivity, specificity, accuracy and area under the curve (AUC) are reported with relevant 95\% CIs. Statistical significance was defined at the two-sided 0.05 level.

\section{RESULTS}

Baseline characteristics and pulmonary function data are summarized in Table 1. Of 59 patients (27 male), 31 (53\%) had OSA. The mean forced expiratory volume in $1 \mathrm{~s}\left(\mathrm{FEV}_{1}\right)$ was $46 \%$ of predicted (range $21 \%$ to $74 \%$ of predicted) and $52 \%$ of patients were on LTOT (oxygen was prescribed and used $18 \mathrm{~h}$ to $24 \mathrm{~h}$ per day) at rates ranging from $1 \mathrm{~L} / \mathrm{min}$ to $4 \mathrm{~L} / \mathrm{min}$ to help maintain saturations $\geq 92 \%$. There were no significant differences in nocturnal saturation profiles between those diagnosed with OSA and those without OSA (Table 2).

Of the pulse oximetry tracings reviewed using the criteria, $11 \mathrm{sub}$ jects were excluded. When examining the capacity of the criteria to detect OSA with PSG as the gold standard classification, an accuracy of $59 \%$ was achieved, with a sensitivity and specificity of $59 \%$ and $60 \%$, respectively. The ROC analysis yielded an AUC of 0.57 (95\% CI 
TABLE 1

Baseline characteristics and pulmonary function

\begin{tabular}{|c|c|c|}
\hline Characteristic & OSA $(n=31)$ & Non-OSA $(n=28)$ \\
\hline Age, years & $69.3 \pm 12.6$ & $65.0 \pm 9.4$ \\
\hline Male sex, n (\%) & $19(46)$ & $8(44)$ \\
\hline Body mass index, $\mathrm{kg} / \mathrm{m}^{2}$ & $36.4 \pm 9.4$ & $37.5 \pm 12.8$ \\
\hline $\mathrm{FEV}_{1}, \mathrm{~L}$ & $1.1 \pm 0.6$ & $1.1 \pm 0.5$ \\
\hline $\mathrm{FEV}_{1}, \%$ predicted & $46.8 \pm 14.9$ & $44.7 \pm 14.2$ \\
\hline FVC, L & $1.7 \pm 0.7$ & $1.9 \pm 0.9$ \\
\hline FVC, \% predicted & $53.6 \pm 14.0$ & $57.9 \pm 21.6$ \\
\hline $\mathrm{FEV}_{1} / \mathrm{FVC}$ & $62.4 \pm 24.2$ & $55.0 \pm 25.6$ \\
\hline Supplementary $\mathrm{O}_{2}$ therapy, $\%$ & 48 & 50 \\
\hline
\end{tabular}

Data presented as mean $\pm S D$ unless otherwise indicated. FEV 1 Forced expiratory volume in $1 \mathrm{~s}$; FVC Forced vital capacity; OSA Obstructive sleep apnea

\section{TABLE 2}

Pulse oximetry characteristics for obstructive sleep apnea (OSA) and non-OSA subjects

\begin{tabular}{lcc}
\hline Measurement & $\begin{array}{c}\text { OSA } \\
(\mathbf{n}=\mathbf{3 1})\end{array}$ & $\begin{array}{c}\text { Non-OSA } \\
(\mathbf{n}=\mathbf{2 8})\end{array}$ \\
\hline Oxygen saturation, \% & $89.2 \pm 3.8$ & $91.0 \pm 4.4$ \\
Highest oxygen saturation, \% & $97.6 \pm 2.4$ & $96.9 \pm 4.7$ \\
Lowest oxygen saturation, \% & $66.7 \pm 15.7$ & $72.1 \pm 16.2$ \\
High desaturation, \% & $90.2 \pm 6.9$ & $92.4 \pm 4.7$ \\
Low desaturation, \% & $85.2 \pm 4.3$ & $86.0 \pm 4.8$ \\
Desaturation events, $n$ & $204.3 \pm 180.6$ & $203.2 \pm 257.0$ \\
Desaturation, events/h & $27.0 \pm 21.9$ & $24.0 \pm 26.4$ \\
Oxygen desaturation events $<90 \%, n$ & $204.3 \pm 180.6$ & $203.2 \pm 257.0$ \\
\% of time $<90 \%$ oxygen saturation & $45.2 \pm 30.5$ & $30.6 \pm 29.0 *$ \\
\hline
\end{tabular}

Data presented as mean $\pm S D$. ${ }^{*}$ Comparison t test $P=0.06$

0.55 to 0.59 ). The ROC analysis was performed for each component of the interpretation criteria and the standard oximetry variables (Table 3 ). When using an ODI with a cut-off $\geq 15$ events/h, the sensitivity and specificity were $60 \%$ and $63 \%$, respectively, and AUC 0.64 (95\% CI 0.62 to 0.66 ) (ROC curve presented in Figure 4). A subgroup analysis of oximetry tracings performed in patients undergoing LTOT and in patients on room air was analyzed separately; the ROC analyses were similar (Table 3). Accuracy for interpretation of tracings on room air was $67 \%$, while only $57 \%$ accuracy was achieved for the tracings from oxygen-dependent patients. No important differences were observed between the OSA and non-OSA groups with regard to pulmonary function, pulse oximetry (Table 2 ) and event and pattern count (data not shown). The OSA group spent a greater mean percentage of nocturnal time below $90 \%$ oxygen saturation; however, this did not reach statisitical significance $(\mathrm{P}=0.06)$ (Table 2$)$.

\section{DISCUSSION}

In the present study, we demonstrated that an explicit approach to interpretation of pulse oximetry tracings can provide a modestly reliable strategy for diagnosis or selection of patients who would most benefit from further polysomnographic assessment for sleep-related breathing disturbances. An interpretation tool, as presented herein, uses observations of predefined desaturation 'events' as well as 'patterns' of oxygen desaturation/resaturation. These visual criteria, in concert with the average oxygen saturation during these patterns, have modest diagnostic accuracy to identify patients who may have OSA. The interpretation criteria enabled us to correctly identify OSA in $55 \%$ of individuals with diagnosed OSA on PSG and to exclude $64 \%$ without a diagnosis OSA - an overall test accuracy of 59\%. This result translated into a detection test with a similar rate for sensitivity and specificity of $60 \%$. When we used an ODI generated by the software, the test became slightly more robust but nonetheless modest in its performance, with a $61 \%$ chance of correct detection.
TABLE 3

The performance of criteria and its components to identify obstructive sleep apnea (OSA)

\begin{tabular}{lccc}
\hline & Sensitivity, \% & Specificity, \% & AUC (95\% CI) \\
\hline Criteria & 59 & 60 & $0.57(0.55-0.59)$ \\
Patterns & 50 & 47 & $0.49(0.46-0.51)$ \\
Events & 58 & 55 & $0.59(0.57-0.61)$ \\
$\mathrm{SaO}_{2}$ & 57 & 59 & $0.55(0.53-0.57)$ \\
$\mathrm{ODI}^{2}$ & 60 & 63 & $0.64(0.62-0.66)$ \\
$\mathrm{RA}$ tracings & 67 & 67 & $0.59(0.55-0.63)$ \\
$\mathrm{O}_{2}$ tracings & 59 & 62 & $0.61(0.56-0.65)$ \\
\hline
\end{tabular}

$O D I$ Oxygen desaturation index; $\mathrm{O}_{2}$ Ability of criteria to detect OSA in the 30 patients using their usual long-term oxygen therapy; RA Ability of criteria to detect OSA in 29 patients on room air; $\mathrm{SaO}_{2}$ Arterial oxygen saturation

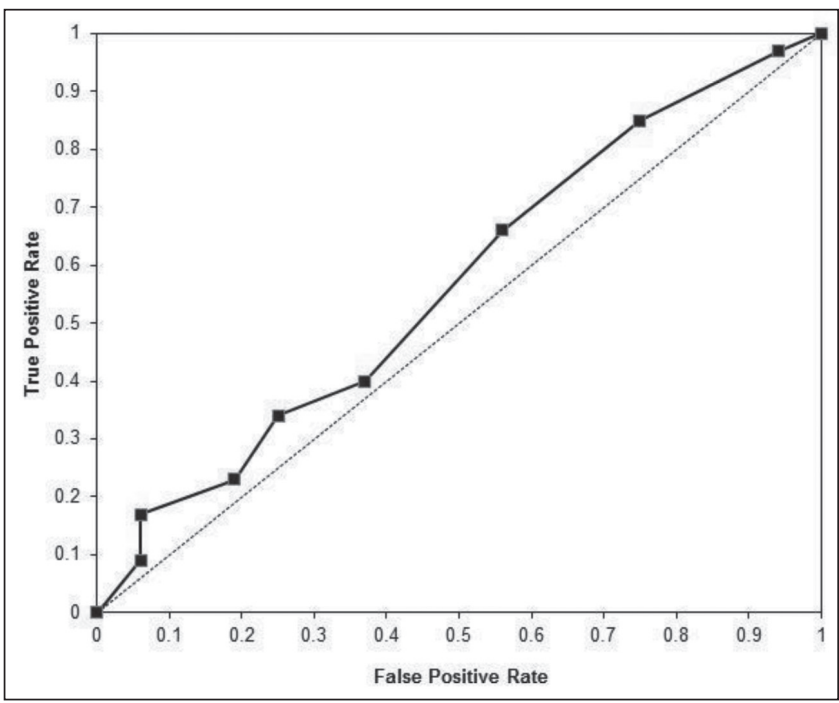

Figure 4) ROC curve of oxygen desaturation index in predicting obstructive sleep apnea. The hash line indicates a hypothetical test equal to random guessing

One novel aspect of the present study was that patients who underwent nocturnal oximetry while on oxygen were included in the analysis. When the oxygen and room air interpretations were analyzed separately, the accuracy was higher in the room air set of tracings; however, overall, the criteria performed similarly in both test groups, with an AUC of approximately 0.6 . It may be expected that supplemental oxygen would limit the diagnostic value of this test; however, because both subsets performed similarly, it may be explained that significant OSA inhibits the adequate passage of low-flow oxygen in these patients with severely diminished pulmonary function. The present study was also unique in that interpretation criteria were based on examining the recording in gross detail using larger time scales $(1 \mathrm{~h}$ and $2 \mathrm{~h})$ as well as capturing more subtle changes through a $15 \mathrm{~min}$ time scale.

In the past two decades, there have been $>20$ articles published attempting to define criteria to detect sleep-related breathing disturbances, such as OSA, from pulse oximetry tracings in primarily nonCOPD populations. In a review by Netzer et al (11), 11 studies were identified and diverse methodologies for oximetry interpretation were reported where available. The contingency analyses from these studies resulted in sensitivity and specificity ranging between $31 \%$ and $91 \%$, and $41 \%$ to $100 \%$, respectively. It should be noted that these studies involved adults with suspected sleep disorders and not specific to COPD. Only one study included pulmonary disease-specific nocturnal oximetry interpretation (12). In this small study, there were eight participants with COPD, three with restrictive lung disease and 15 with OSA syndrome. In this study, using a mathematical interpretation of the $\mathrm{SaO}_{2}$ signal the number of apneas detected by PSG and the 
calculated desaturation index from oximetry were correlated $\left(\mathrm{r}^{2}=0.92\right.$; $\mathrm{P}<0.01$ ). Other authors have been successful using similar methodology to the present study in patients with congestive heart failure. Sériès et al (13) examined home nocturnal oximetry tracings for detection of sleep-related breathing disturbances in patients with chronic heart failure $(n=50)$ and reported their analysis to be sensitive $(85 \%)$ and specific (93\%) for identifying OSA. A smaller study involving congestive heart failure patients $(n=10)$ who were examined for ventilatory disturbances, such as Cheyne-Stokes breathing, concluded that pulse oximetry was effective in identifying sleep-related breathing changes, and was simple, inexpensive and well tolerated (14). Heart failure and COPD can coexist in patients with overlap of symptoms, thereby complicating diagnosis and treatment (15).

The coexistence of both OSA and COPD has been termed the 'overlap syndrome' (16). This occurs in approximately $5 \%$ to $10 \%$ of the population with obstructive airways disease, which represents approximately $0.5 \%$ to $1 \%$ of the general population $\geq 40$ years of age (17). When the overlap syndrome has been identified, it has been found to be associated with poorer quality of sleep, lower quality of life, increased morbidity and increased mortality $(17,18)$. It is clinically worthwhile to identify, as early as possible, this subpopulation of patients with COPD given the interventions that exist and have proven beneficial (18). However, both diseases share common symptoms such as fatigue and nonrestorative sleep. Although clinicians may currently use the common indicators of disruptive snoring, excessive daytime sleepiness and an elevated body mass index to suspect OSA in a patient with COPD, many patients with OSA do not have these characteristics $(18,19)$.

In this clinical setting, this becomes highly evident and problematic when we consider the variation in physician interpretation of nocturnal oximetry. A recent study addressed this question and revealed the wide inconsistency of physician opinions concerning the utility of oximetry and measurements they believed to be pertinent to the diagnosis and management of their patients (20). All subjects (pulmonary physicians) were asked to interpret three oximetry tracings and provide a diagnosis; approximately one-half of the physicians were able to. Diagnoses varied widely including sleep-related breathing disturbances, lung or cardiac disease, rapid eye movementassociated desaturation and neuromuscular disease. Management recommendations also varied among the physicians: the top three were PSG, nocturnal oxygen prescription and the need for more medical information. The top four physician-selected oximetry data chosen for relevancy included $\mathrm{SpO}_{2}$ waveform and pattern. Some other variables not measured by the oximetry that were considered to be important for interpretation were whether they had witnessed apneas, patient had known heart or lung disease, recording length and sleepiness status.

There have been several published methods regarding mathematical models to predict OSA from indexes of nocturnal pulse oximetry. An aggregated model developed a predictive calculation that was able to accurately detect OSA based on differences in saturation over $12 \mathrm{~s}$ intervals (delta index), desaturation events and data on time spent below various saturation thresholds (21). The main finding was that combining the variables of oximetry improved the precision of OSA diagnosis compared with using the delta index or desaturation indexes alone $\left(r^{2}=0.70\right)$. This method was validated in a prospective cohort whereby the oximetry data were collected simultaneously during PSG and separated for independent analyses. Recently, an analysis was performed to evaluate four different statistical pattern recognition techniques of $\mathrm{SpO}_{2}$ signals to circumvent the need for PSG in the detection of OSA in otherwise normal patients (22). The most accurate classifier was derived by a linear discriminant analysis algorithm with spectral features (accuracy $87.6 \%$ and AUC 0.925). In contrast, a testing of two predictive models that included integration of a clinical score, pulmonary function results, arterial blood gas data and nocturnal pulse oximetry interpretation failed to be valid when used in a prospective population that was similar and selected in the same way (23). Other predictive methods used in patient populations with suspected OSA that integrated clinical features and/or questionnaires as well as oximetry data were found to have modest capacity for identifying OSA, with some success at eliminating patients without OSA as well as categorizing high-risk patients needing further investigation $(24,25)$.

A limitation of the present study was that the oximetry interpretation analysis was performed retrospectively and would need to be validated prospectively to confirm its utility. The study included a limited number of subjects and would need to be further explored with a larger subset of patients. However, the 95\% CIs for all the explicit oximetry criteria were relatively narrow, spanning $\leq 8 \%$ and excluded AUC $\geq 0.70$. The subjectivity of the analytical method must also be considered when selecting a strategy for oximetry tracing interpretation and may warrant adequate training for those who use nocturnal oximetry in their management of patients with potential sleep-related breathing disturbances; however, our tracing visual analysis criteria were explicitly defined and demonstrated inter-rater concordance $>80 \%$ before beginning the analysis of our patients with COPD.

Although our study appears to support the findings outlined in practice parameters for the indications for PSG, which states that oximetry lacks the specificity and sensitivity to be used as an alternative to PSG for diagnosing sleep-related breathing disorders (26), it does warrant further investigation. There remains an important need to use simplified diagnostic techniques for patients with severe COPD. Our data suggest that simple pulse oximetry appears to be neither sensitive nor sufficiently specific to stand alone as a diagnostic procedure. There appears to be more promise in the use of mathematical waveform interpretation rather than visual waveform analyses to risk stratisfy patients with suspected sleep-related breathing disturbances such as OSA. This has yet to be tested in patients with advanced COPD.

ACKNOWLEDGEMENTS: The authors thank Ryan Chan, an MSc candidate from the School of occupational and Physical Therapy at McGill University for his assistance in protocol development and data collection. AS and MB designed the research study, analyzed the data and wrote the manuscript. AS performed the research. NW reviewed the data and contributed to the writing and editing of the manuscript. The authors also acknowledge the ongoing support of the research fund at Mount Sinai Hospital Center.

\section{APPENDIX 1: NOCTURNAL OXIMETRY TRACINGS}

\section{Protocol for the analysis and interpretation criteria}

Gross exclusion:

- In a 1-hour time scale

1) Exclude subject from analysis if $\geq 20 \%$ of the total tracing time contains breaks.

2) A section of the tracing can be excluded from analysis if within a 30 minute period (in a 2 hour time scale), there are $\geq 6$ breaks OR if there is no discernable pattern.

Events (within 2 hour time scale):

- A change in the tracing defined by the following:

- Average desaturation of $\geq 4 \%$ from the baseline average

- Minimal duration of 30 minutes with similar pattern magnitude/frequency.

- A second event can occur if during an event there is a progressive change in mean desaturation of $8 \%$ or greater from baseline.

Patterns (within 15 minute time scale):

(Must meet all of the criteria)

- Exclusion: Within a 5 minute period, there are $\geq 3$ breaks.

- Pattern: A rise and fall of at least 3 similar saturation cycles 
(Note the mean saturation of the 3 or more cycles)

- Saturation pattern must last at least 4 minutes. If the mean saturation changes and remains constant for $\geq 8$ minutes, note down the new mean.

- The pattern amplitude must be at least 3\%

- The peak or nadir of the pattern must be similar in terms of shape
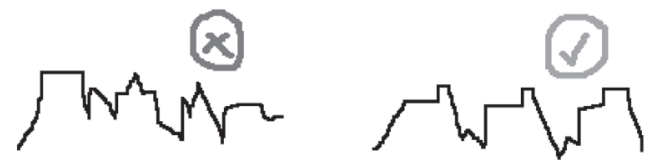

- Changes in saturation range (max-min) or average saturation are not considered a change in pattern unless it is paired with a significant change in frequency
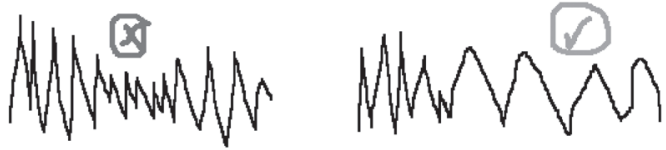

- A significant drop or change in saturation is considered a change in pattern only if it lasts more than 10 minutes or meets the aforementioned patterning criteria

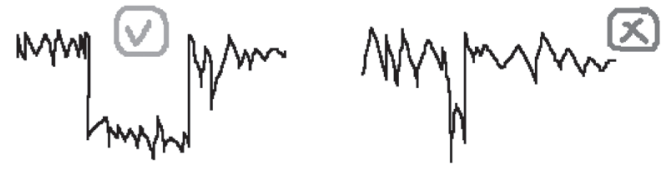

OSA diagnosis:

- A case is identified as OSA if the following criteria are met:

1. A mean $\mathrm{SpO} 2 \leq 90 \%$

2. At least one of the following:

- \# of events $\geq 4$

- \# of patterns $\geq 12$

- A case is identified as no-OSA if the previous criteria are not met.

\section{REFERENCES}

1. Mohsenin V. Sleep in chronic obstructive pulmonary disease. Semin Respir Crit Care Med 2005;26:109-16.

2. Kutty K. Sleep and chronic obstructive pulmonary disease. Curr Opin Pulm Med 2004;10:104-12.

3. Krachman S, Minai OA, Scharf SM. Sleep abnormalities and treatment in emphysema Proc Am Thorac Soc 2008;5:536-42.

4. Tirlapur VG, Mir MA. Nocturnal hypoxemia and associated electrocardiographic changes in patients with chronic obstructive airways disease. N Engl J Med 1982;306:125-30.

5. Chaouat A, Naeije R, Weitzenblum E. Pulmonary hypertension in COPD. Eur Respir J 2008;32:1371-85.

6. Jelic S. Diagnostic and therapeutic approach to coexistent chronic obstructive pulmonary disease and obstructive sleep apnea. Int J Chron Obstruct Pulmon Dis 2008;3:269-75.
7. Machado MC, Vollmer WM, Togeiro SM, et al. CPAP and survival in moderate-to-severe obstructive sleep apnoea syndrome and hypoxaemic COPD. Eur Respir J 2010;35:132-7.

8. American Thoracic Society. Indications and standards for cardiopulmonary sleep studies. Am Rev Respir Dis 1989;139:559-68.

9. The AASM Manual for the Scoring of Sleep and Associated Events: Rules, Terminology and Technical Specifications, 1st edn. Westchester: American Academy of Sleep Medicine, 2007.

10. Eng J. ROC analysis: Web-based Calculator for ROC Curves. Baltimore: Johns Hopkins University [updated 2006 May 17; cited 2011 August 2]. <www.jrocfit.org> (Accessed September 2010).

11. Netzer N, Eliasson AH, Netzer C, Kristo DA. Overnight pulse oximetry for sleep-disordered breathing in adults: A review. Chest 2001;120:625-33.

12. Pépin JL, Lévy P, Lepaulle B, Brambilla C, Guilleminault C. Does oximetry contribute to the detection of apneic events? Mathematical processing of the $\mathrm{SaO}_{2}$ signal. Chest 1991;99:1151-7.

13. Sériès F, Kimoff RJ, Morrison D, et al. Prospective evaluation of nocturnal oximetry for detection of sleep-related breathing disturbances in patients with chronic heart failure. Chest 2005;127:1507-14.

14. Resta O, Foschino-Barbaro MP, Bonfitto PG, et al. Nocturnal oxygen desaturation in patients with congestive heart failure. Boll Soc Ital Biol Sper 1999;75:31-8.

15. Hawkins NM, Petrie MC, Jhund PS, Chalmers GW, Dunn FG, McMurray JJ. Heart failure and chronic obstructive pulmonary disease: Diagnostic pitfalls and epidemiology. Eur J Heart Fail 2009;11:130-9.

16. Flenley DC. Sleep in chronic obstructive lung disease. Clin Chest Med 1985;6:51-61.

17. Weitzenblum E, Chaouat A, Kessler R, Canuet M. Overlap syndrome: Obstructive sleep apnea in patients with chronic obstructive pulmonary disease. Proc Am Thorac Soc 2008;5:237-41.

18. Owens RL, Malhotra A. Sleep-disordered breathing and COPD: The overlap syndrome. Respir Care 2010;55:1333-44.

19. Sanders MH, Newman AB, Haggerty CL; Sleep Heart Health Study. Sleep and sleep-disordered breathing in adults with predominantly mild obstructive airway disease. Am J Respir Crit Care Med 2003;167:7-14.

20. Ramsey R, Mehra R, Strohl KP. Variations in physician interpretation of overnight pulse oximetry monitoring. Chest 2007;132:852-9.

21. Magalang UJ, Dmochowski J, Veeramachaneni S, et al. Prediction of the apnea-hypopnea index from overnight pulse oximetry. Chest 2003;124:1694-701.

22. Marcos JV, Hornero R, Alvarez D, del Campo F, Zamarrón C. Assessment of four statistical pattern recognition techniques to assist in obstructive sleep apnoea diagnosis from nocturnal oximetry. Med Eng Phys 2009;31:971-8.

23. Roche N, Herer B, Roig C, Huchon G. Prospective testing of two models based on clinical and oximetric variables for prediction of obstructive sleep apnea. Chest 2002;121:747-52.

24. Deegan PC, McNicholas WT. Predictive value of clinical features for the obstructive sleep apnoea syndrome. Eur Respir J 1996;9:117-24.

25. Gurubhagavatula I, Maislin G, Pack AI. An algorithm to stratify sleep apnea risk in a sleep disorders clinic population. Am J Respir Crit Care Med 2001;164(10 Pt 1):1904-9.

26. Kushida CA, Littner MR, Morgenthaler T, et al. Practice parameters for the indications for polysomnography and related procedures: An update for 2005. Sleep 2005;28:499-521. 


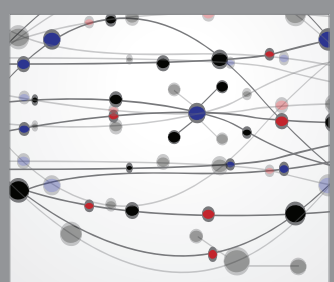

The Scientific World Journal
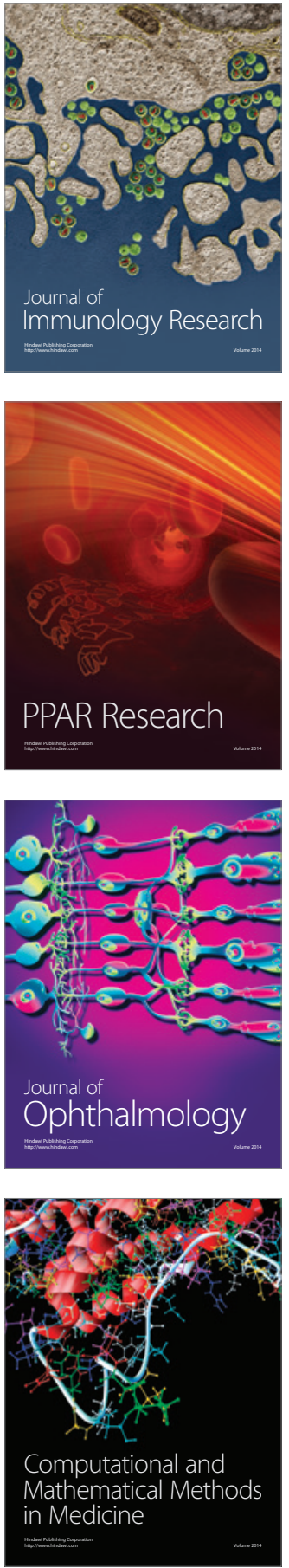

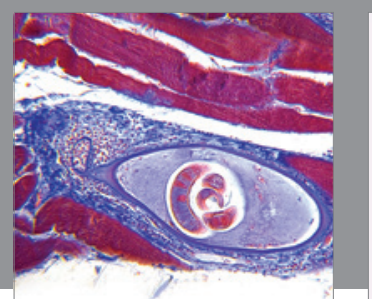

Gastroenterology Research and Practice

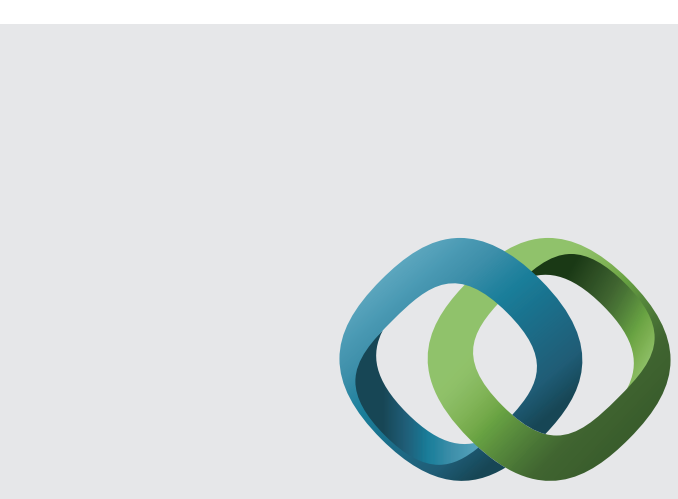

\section{Hindawi}

Submit your manuscripts at

http://www.hindawi.com
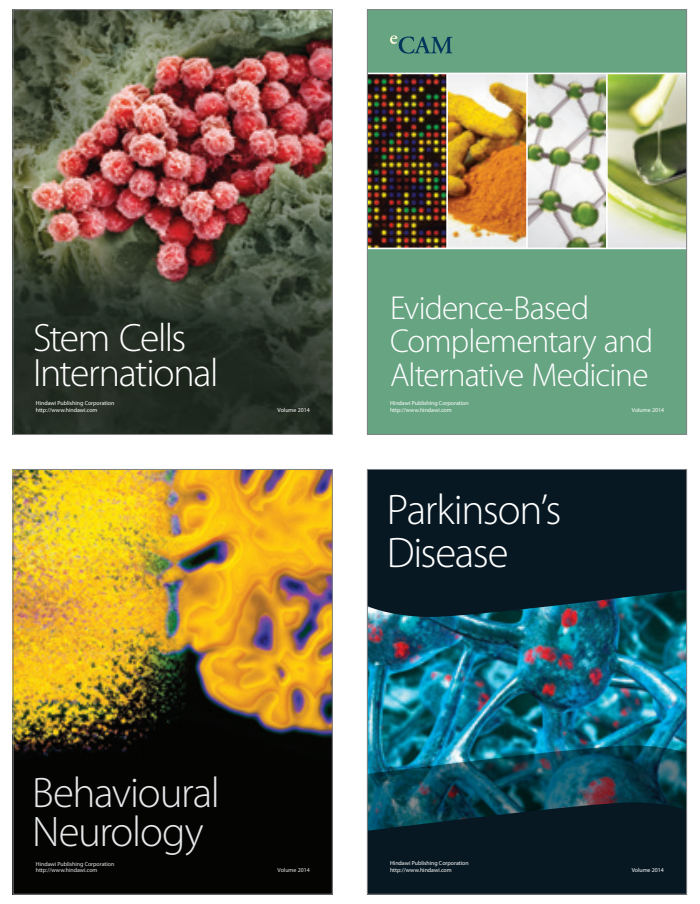
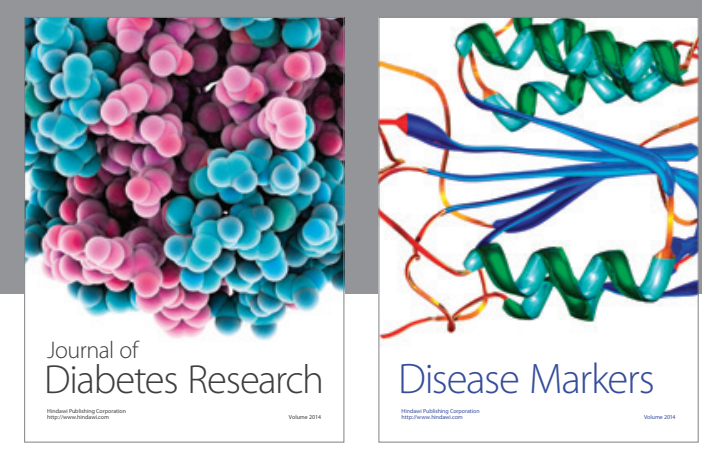

Disease Markers
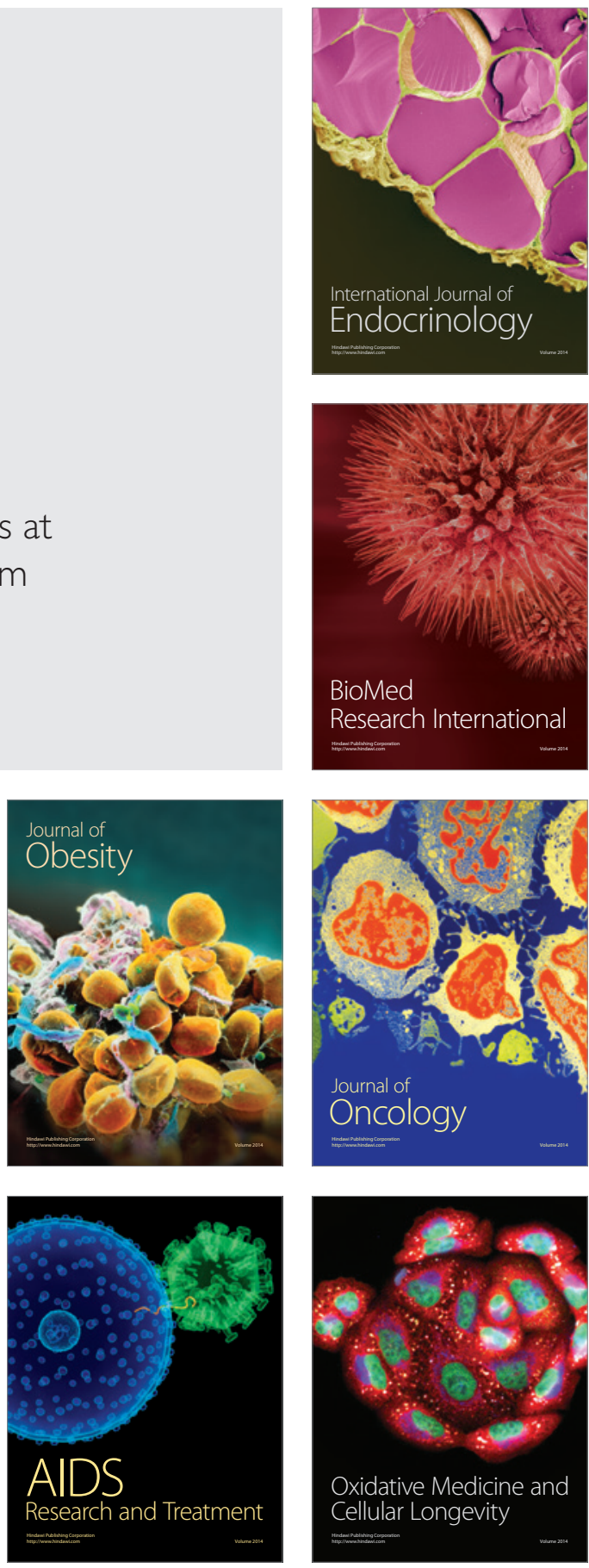